\title{
Quantum Nondemolition Measurement of a Kicked Qubit
}

\author{
Andrew N. Jordan and Markus Büttiker \\ Département de Physique Théorique, Université de Genève, CH-1211 Genève 4, Switzerland
}

(Dated: June 22, 2004)

\begin{abstract}
We propose a quantum nondemolition measurement using a kicked two-state system (qubit). By tuning the waiting time between kicks to be the qubit oscillation period, the kicking apparatus performs a nondemolition measurement. While dephasing is unavoidable, the nondemolition measurement can (1) slow relaxation of diagonal density matrix elements, (2) avoid detector back-action, and (3) allow for a large signal-to-noise ratio. Deviations from the ideal behavior are studied by allowing for detuning of the waiting time, as well as finite-time, noisy pulses. The scheme is illustrated with a double-dot qubit measured by a gate-pulsed quantum point contact.
\end{abstract}

PACS numbers: 05.45.Mt,03.67.Lx,03.65.Wj,73.23.-b

\section{INTRODUCTION}

Motivated by general interest in mesoscopic quantum phenomena, as well as by specific interest in quantum computation, there has been intensive experimental and theoretical effort to study coherence properties and measurement possibilities of solid state qubits. In particular, there has been great interest in different quantum measurement proposals. This paper is concerned with a type of weak measurement, referred to as a Quantum Nondemolition (QND) measurement. QND measurements are important when one wishes to extract information about a quantum state over a period of time without destroying it. This type of measurement has been proposed and im-

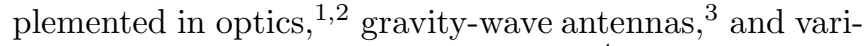
ous realizations of harmonic oscillators ${ }^{4}$. QND measurements have also been proposed as a quantum computing measurement method for single spins..$^{5.6}$

A weak measurement of a quantum system occurs when the state of the system is not perfectly correlated with the detector, in contrast to the usual projective measurement. Weak measurement has an advantage over projective measurement in that one can study the coherent oscillations of a single quantum state over a long time period, whereas with projective measurements, one must repeat the measurement many times to see the quantum coherent oscillations. However, weak measurements have the disadvantage that they usually act back on the system, so that one is never sure whether the result stems from the original quantum state alone. QND schemes try to overcome this limitation by coupling the detector to the system via an operator that represents an approximate constant of motion of the quantum system. In this way, the detector disturbs the system in a minimal way.

Recently, Averin proposed a QND scheme for a twostate system that involves a time dependent coupling which follows the qubit in spin-space while weakly measuring it $\underline{7}$ The scheme was illustrated with a Josephsonjunction system as an example of a mesoscopic qubit. The purpose of this paper is to generalize Averin's qubit nondemolition measurement. The method we propose is quite general and could be implemented in many different detector realizations. The basic idea is that rather than

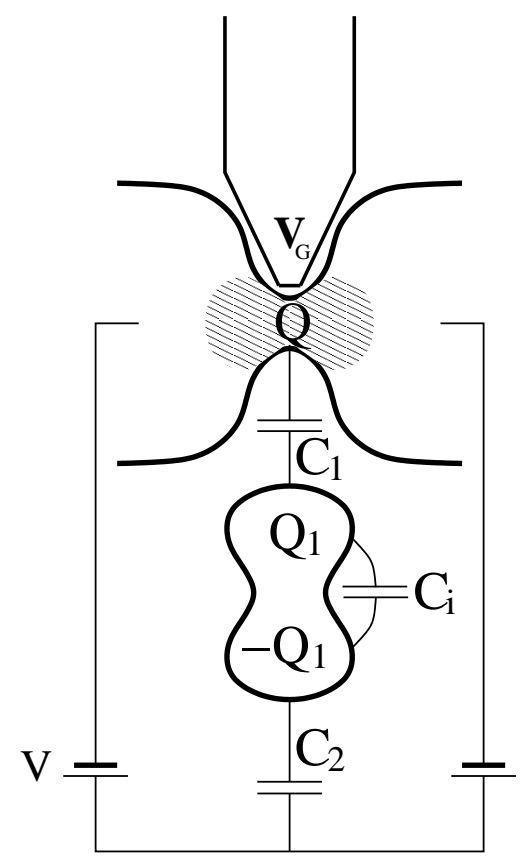

FIG. 1: The quantum nondemolition measurement performed with a quantum point contact. A double-dot with charge $Q_{1}$ is capacitively coupled to a quantum point contact. Current through the quantum point contact is periodically switched on and off with the help of a gate voltage $V_{G}$ with the waiting time between pulses given by the Rabi oscillation period of the double dot.

try to chase the spin around in spin space, we let it come to us. This is done by strobing the qubit/detector coupling constant in time, so the detector only has access to the qubit at approximately discrete moments. In this reduced problem, by fixing the waiting time between pulses to be a multiple of the qubit oscillation time $\tau_{q}$, the observable we want to measure is static in time. Thus, by measuring over many pulses, a large (back-action free) signal-to-noise ratio may be obtained. The liability of this proposal is that one cannot detect what is happening while the coupling is turned off, so the qubit must have a reasonably high quality factor. However, this scheme has several advantages over the original proposal: (1) the 
coupling to the qubit needs only one qubit operator (the one we want to measure), (2) an arbitrary qubit Hamiltonian is allowed, (3) in the ideal case, no assumptions need to be made about the density matrix of the qubit, and (4) in the non-ideal case, the pulses may be noisy. These advantages make this kicked QND measurement feasible with existing technology.

Although kicking the qubit in time sounds rather violent, it is in fact coherent because we choose the pulses to be fast, so they may be described by a series of delta function kicks. Dephasing is induced by the fluctuating measurement apparatus. The inspiration for the present paper is from a venerable model of quantum chaos, the kicked rotor ${ }^{8.9}$ Here, the situation is even simpler because the kicked quantum system has only two states. Nevertheless, it is the play-off between the waiting time between kicks and the system dynamics that yields the interesting physics. In this way, the kicked QND measurement is similar to kicked atoms which give rise to accelerator modes 10 or quantum resonances $\stackrel{11}{1}$ However, the idea of "stroboscopic measurement" is actually quite old, and the basic idea appears already for harmonic oscillators in Refs. 12 13, and has recently been proposed for the nanomechanical resonator 14

An implementation of the quantum nondemolition experiment is shown in Fig. 1. A double quantum-dot representing a charge qubit is capacitively coupled to a quantum point contact (QPC). The QPC is opened and closed with the help of a plunger gate with voltage $V_{G}$. Before considering this specific physical system, we first present general theoretical considerations. After the detector assumptions are reviewed in Sec. [II the Hamiltonian is introduced and the ideal quantum nondemolition measurement is described in Sec. III] Deviations are then allowed in Secs. IV and $\nabla$ with detuning between the detector pulses and the qubit oscillation time, as well as noisy pulses of finite width. We then describe the details of the quantum double-dot measured by the QPC in Sec. VI (see Fig. 1). Sec. VII contains our conclusions, and Appendix $\mathrm{A}$ contains our perturbation method.

\section{DETECTOR ASSUMPTIONS}

A realistic, effective detector has two necessary characteristics: the measurement output should behave classically and the response time of the detector should be much shorter than the period of the qubit oscillations. As mentioned in the introduction, there can be strong detectors whose states are well correlated with the state of the system and thus make a projective measurement of the qubit's state, or there can be weak measurement which only extracts partial information about the quantum state. For the weak measurement, we assume that linear response applies ${ }^{15}$ Quantitatively, if the bare detector variable is $f$, (which we treat as a classical random variable), coupled to the qubit with the operator $\sigma_{z}$, and the detector output is $\mathcal{O}$, then it is given by a noisy, random part $q$, plus a small component that reflects the dynamics of the qubit

$$
\mathcal{O}(t)=q(t)+\frac{\lambda}{2} \sigma_{z}(t)
$$

where $\lambda$ is the response coefficient which indicates how sensitive the detector is, and $\sigma_{z}$ is the qubit operator that we are trying to measure. With these assumptions, the temporal correlation function of the detector's output is

$$
\begin{aligned}
C(t) & =\langle\mathcal{O}(\tau+t) \mathcal{O}(\tau)\rangle_{\tau}=2 \pi S_{q} \delta(t-0)+\frac{\lambda^{2}}{2} K(t), \\
K(t) & =(1 / 2) \operatorname{Tr}\left[\rho \sigma_{z}(t) \sigma_{z}(0)\right]
\end{aligned}
$$

where $S_{q}$ is the spectral weight of the noise $q$, the density matrix of the qubit is $\rho$, and we have made use of the fact that the detector response time is much faster than the qubit oscillation time. Cross terms of the form $\left\langle q \sigma_{z}\right\rangle$ vanish because the detector noise is uncorrelated with the qubit oscillations. The spectral density of the detector is now given by

$$
S(\omega)=\int_{-\infty}^{\infty} \frac{d t}{2 \pi} e^{i \omega t} C(t)=S_{q}+\frac{\lambda^{2}}{2} \int_{-\infty}^{\infty} \frac{d t}{2 \pi} e^{i \omega t} K(t) .
$$

This is the object that is experimentally accessible for low frequencies. If the detector is coupled to the qubit statically, over several oscillations the measurement device will measure the desired quantum operator $\left(\sigma_{z}\right)$, but there will be detector back-action altering the qubit dynamics. This back-action imposes a fundamental limit on the signal-to-noise ratio of 4.17 We turn now to the pulsed scheme to overcome this limitation.

Instead of following the spin, we let it complete the Rabi oscillation and couple only after a full rotation. The time scale of the on/off transition should be slow to the detector (so linear response still applies), but fast to the qubit. If the effective qubit has higher energy states, then the time scale of the kick must also be sufficiently slow so as not to excite these states. The dynamic coupling will result in a ladder of pulses at the measurement output device, typically of height $\lambda$. The measurement of the quantum state of the qubit is extracted by examining how the rungs of the ladder are correlated with each other above the white noise background.

\section{THE IDEAL SCENARIO}

We first consider the simplest case where the duration of the pulse is zero. The qubit is then coupled to the detector by periodic delta functions in time of waiting time $\tau$, with $f$ describing the random detector variable. The Hamiltonian for this ideal scenario is given by

$$
H=\frac{\epsilon}{2} \sigma_{z}+\frac{\Delta}{2} \sigma_{x}+\sum_{n=-\infty}^{\infty} \delta(t-n \tau) \frac{\sigma_{z} f(t)}{2}+H_{f},
$$


where $H_{f}$ is the detector Hamiltonian. The evolution operator corresponding to this time-dependent Hamiltonian may be found from

$$
\mathcal{U}^{t}=\mathrm{T} \exp \left[-i \int_{0}^{t} d t^{\prime} H\left(t^{\prime}\right)\right]
$$

where $\mathrm{T}$ is the time ordering operator. The time integration gives the free propagator when the delta function is zero, while during the kick, we can integrate the delta function over a small time interval $\varepsilon$ to find the qubit propagator for the kick:

$$
\begin{aligned}
\lim _{\varepsilon \rightarrow 0} & \exp \left\{-i \int_{\tau-\varepsilon}^{\tau+\varepsilon} d t^{\prime}\left[H_{q}+\delta(t-\tau) \frac{\sigma_{z} f(t)}{2}\right]\right\} \\
& =\exp \left[-i \sigma_{z} f(\tau) / 2\right]
\end{aligned}
$$

The contribution of the free propagator is proportional to $\varepsilon$ in this interval so it vanishes when we take $\varepsilon \rightarrow 0$, while the kick contribution stays finite. This allows us to factorize the propagator as

$$
\begin{aligned}
\mathcal{U}_{i} & =\mathcal{U}_{\mathcal{F}} \mathcal{U}_{\mathcal{K}}, \quad \mathcal{U}_{\mathcal{F}}=\exp \left[-i \tau(\epsilon / 2) \sigma_{z}-i \tau(\Delta / 2) \sigma_{x}\right] \\
\mathcal{U}_{\mathcal{K}} & =\exp \left[-i \sigma_{z} f\left(\tau_{i}\right) / 2\right], \quad \mathcal{U}^{t}|\psi\rangle=\prod_{i=1}^{n} \mathcal{U}_{i}|\psi\rangle
\end{aligned}
$$

Here, $n=t / \tau$ is the discrete time index. It is important to note that $\mathcal{U}_{i}$ changes during each time step because $f$ has its own dynamics while the coupling is turned off that is governed by $H_{f}$. So far, the waiting time interval $\tau$ may be arbitrary. In the ideal case, we choose $\tau$ to be $\tau_{q}=4 \pi / \sqrt{\epsilon^{2}+\Delta^{2}}$, the oscillation period of the qubit. Then, the free propagator is simply the identity matrix, so the total propagator is composed of $\mathcal{U}_{\mathcal{K}}$ 's only.

Now that we have the propagator, all dynamical properties of the qubit may be investigated. The first important property is that of the coherence of the qubit. Decoherence effects are caused by the randomly changing $f$ variable. If we begin kicking with an initially pure state $\psi=(\alpha, \beta)$ in the $z$ basis, the density matrix changes in time as

$$
\rho(n)=\left(\begin{array}{cc}
|\alpha|^{2} & Z \alpha \beta^{*} \\
Z^{*} \alpha^{*} \beta & |\beta|^{2}
\end{array}\right),
$$

where $Z=\prod_{i=1}^{n} e^{i f_{i}} \rightarrow \exp \left[i n\langle f\rangle-n\left\langle\delta f^{2}\right\rangle / 2\right]$ by the central limit theorem. Here, we see explicitly that the pulsed coupling keeps the diagonal matrix elements constant in time, but there is exponential dephasing controlled by the fluctuations in $f$ whose dephasing rate is simply the noise of the detector variable. It is clear that information about the $x$ and $y$ variables is being destroyed by the measuring apparatus, while we have carefully preserved the $z$ information of the state.

One simple but important quantity to calculate is the expectation value of $\sigma_{z}(n)$,

$$
\left\langle\sigma_{z}(n)\right\rangle=\operatorname{Tr}\left[\rho \mathcal{U}^{t} \sigma_{z} \mathcal{U}^{t^{\dagger}}\right]
$$

For the ideal case, the propagator is just a function of $\sigma_{z}$, so $\sigma_{z}$ is a constant of motion. $\operatorname{Tr}\left[\rho \sigma_{z}\right]=|\alpha|^{2}-|\beta|^{2}$, so the expectation value is a measure of the population that is time independent. We may now calculate the time correlation function of the detector output. The discrete version of Eq. (2) is

$$
C(n)=2 \pi S_{q} \delta_{n, 0}+\left(\lambda^{2} / 2\right) \operatorname{Tr}\left[\rho \sigma_{z} \sigma_{z}(n)\right]
$$

where $\sigma_{z}(n)=\mathcal{U}^{-n} \sigma_{z} \mathcal{U}^{n}$. Again, $\sigma_{z}$ commutes with $\mathcal{U}$, $\sigma_{z}^{2}=1$ and $\operatorname{Tr} \rho=1$, so $K(n)$ never decays with $n$, because every rung on the signal ladder is identical. If we calculate the discrete version of the spectral function, Eq. (3), the sum diverges at $\omega=0$. This tells us that the accumulated signal about $\sigma_{z}$ may be arbitrarily larger than the noise of the detector, $S_{q}$, regardless of how small the response coefficient $\lambda$ is.

\section{DETUNING}

We now generalize this ideal behavior to more realistic situations. In this section, we let the waiting time $\tau$ be close to the qubit oscillation time, but not equal. This gives us a perturbation parameter $\gamma$, where $\tau / \tau_{q}=1+\gamma$. We take $\gamma \ll 1$ and let $\rho=(1 / 2) \mathbb{1}$ for calculational simplicity. Noting the identity $\exp [-i(\alpha / 2) \hat{n}$. $\sigma]=\mathbb{1} \cos \alpha / 2-i \hat{n} \cdot \sigma \sin \alpha / 2$ with $\alpha=\tau \sqrt{\epsilon^{2}+\Delta^{2}}$ and the unit vector $\hat{n}$ chosen to give the free propagator $\left(n_{z}=\tau \epsilon / \alpha, n_{x}=\tau \Delta / \alpha\right)$, we choose $\alpha=4 \pi+\gamma$ implying $\mathcal{U}_{\mathcal{F}}=\exp [-i(\gamma / 2) \hat{n} \cdot \sigma]$. This is the same as free evolution by an angle $\gamma$ of our choosing. Time evolution is now different from the ideal case because the free and kick evolution operators do not commute. We may investigate the time correlator of $\sigma_{z}$ by expanding $\mathcal{U}$ to second order in $\gamma$ to find

$$
K(n)=1-\left(\frac{\Delta \tau_{q} \gamma}{2 \pi}\right)^{2}\left(\frac{n}{2}+\operatorname{Re} \sum_{j=1}^{n-1} X_{j}\right)
$$

where the sum describes the interaction with the detector and the $X_{j}$ are recursively defined by:

$$
X_{1}=e^{i f_{1}}, X_{j}=e^{i f_{j}}\left(1+X_{j-1}\right), j=2, \ldots, n-1
$$

The $f_{i}$ are chosen from the same probability distribution and are assumed to be independent, so we can average over the $f$ 's using $\left\langle e^{i f}\right\rangle=\exp [i\langle f\rangle-S / 2]$ where $S=\left\langle\delta f^{2}\right\rangle$ is the dephasing rate found from Eq. (8). The sum in Eq. (11) may now be carried out to yield 


$$
\langle K(n)\rangle=1-\left(\frac{\Delta \tau_{q} \gamma}{2 \pi}\right)^{2}\left(\frac{n}{2}+\frac{e^{-(S / 2)(n+1)}+e^{-S / 2}(n-1)-n e^{-S}}{\left(1-e^{-S / 2}\right)^{2}}\right) .
$$

With the assumption $\gamma \ll 1$, Eq. (13) contains two (dimensionless) rates, the dephasing rate, $S$, and a much smaller "nondemolition" rate. It is this nondemolition rate that controls the decay for times longer than the initial transients, $\langle K(n)\rangle=1-\mathcal{G}_{d} n$,

$$
\mathcal{G}_{d}=\left(\frac{\Delta \tau_{q} \gamma}{2 \pi}\right)^{2}\left(\frac{1}{2}+\frac{1}{e^{S / 2}-1}\right)
$$

Although this represents only the second order contribution in $\gamma$, we can extrapolate to longer time by exponentiating, $\langle K(n)\rangle \approx \exp \left[-\mathcal{G}_{d} n\right]$. Including the noise of the detector $S_{q}$, the correlation function determines the spectral density $S(\omega)=(1 / 2 \pi) \sum_{n=-\infty}^{\infty} C(n) \cos \omega n$ of the detector output. Besides the back-action, there are always other relaxation processes that are not well controlled. We therefore introduce $\mathcal{G}_{e}$ as a phenomenological environmental relaxation rate to find

$$
S(\omega)=S_{q}+\frac{\lambda^{2}}{4 \pi} \frac{\sinh \left(\mathcal{G}_{e}+\mathcal{G}_{d}\right)}{\sin ^{2}(\omega / 2)+\sinh ^{2}\left(\mathcal{G}_{e} / 2+\mathcal{G}_{d} / 2\right)} .
$$

The $\sin ^{2}(\omega / 2)$ reflects a fundamental periodicity in the experiment: the effect is still there if we wait some multiple of the oscillation period. If we further assume that $S \ll 1$ (the detector noise is weak), than we can expand the decay rate $\mathcal{G}_{d}$ and divide by $\tau_{q}$ to obtain the real time decay rate,

$$
\Gamma_{d}=\left(\frac{\Delta \gamma}{2 \pi}\right)^{2} \frac{2 \tau_{q}}{\left\langle\delta f^{2}\right\rangle}
$$

This special case is analogous to Averin's line-shape ${ }^{7}$ The whole point of the scenario is that Eq. (15) describes the natural line-width of the qubit with detector backaction as a tunable parameter.

\section{NOISY PULSES}

In experiments, the ladder of pulses will have finite width and their own noise associated with them. In this section, we take these effects into account. To model this realistic situation, we consider the following Hamiltonian:

$$
H=\frac{\epsilon}{2} \sigma_{z}+\frac{\Delta}{2} \sigma_{x}+\frac{\tau_{q}}{\tau_{G}} U(t) \sigma_{z}+H_{U} .
$$

The variable $U(t)$ generalizes the delta function coupling $f(t) \delta(t-n \tau)$ in Sec. III by broadening it into a pulse that lasts for a short time $\tau_{G}$ with a large prefactor $r=\tau_{q} / \tau_{G}$. While the pulse is on, the detector variable may be decomposed into an average height with fluctuations: $U(t)=U+\delta U(t)$. Like $\langle f\rangle, U$ simply serves to renormalize the $\sigma_{z}$ prefactor, $\bar{\epsilon}=\epsilon+r U$, and will not enter into the final results. The fluctuations are treated in the white noise approximation,

$$
\langle\delta U(t)\rangle=0, \quad\left\langle\delta U(t) \delta U\left(t^{\prime}\right)\right\rangle=\left\langle\delta U^{2}\right\rangle \delta\left(t-t^{\prime}\right) .
$$

Note that although $U(t)$ has units of energy, the convention of Eq. (18) defines $\left\langle\delta U^{2}\right\rangle$ to also have units of energy. Now, we wish to calculate the ensemble averaged correlator $K(t)$, Eq. (2), for one pulse after making the diagonal assumption for the density matrix to simplify the analysis. The method we use is analogous to the discrete case. The equations of motion are straightforward,

$$
\begin{aligned}
& \dot{\sigma}_{x}=-[\bar{\epsilon}+r \delta U(t)] \sigma_{y}, \dot{\sigma}_{y}=[\bar{\epsilon}+r \delta U(t)] \sigma_{x}-\Delta \sigma_{z}, \\
& \dot{\sigma}_{z}=\Delta \sigma_{y} .
\end{aligned}
$$

The time correlator Eq. (2) may be calculated by formally integrating Eqs. (19) in time to a given order $n$, as shown in the appendix. The closed algebra of the Pauli matrices allows the solution to be formally written in terms of $n$ time ordered integrals. The condition $r \gg 1$ selects a certain class of terms in the perturbation theory that may be averaged and summed to all orders yielding

$$
\langle K(t)\rangle=1-\frac{\Delta^{2} t}{\left\langle\delta U^{2}\right\rangle r^{2}}-\frac{\Delta^{2}}{\left(\left\langle\delta U^{2}\right\rangle r^{2}\right)^{2}}\left(e^{-\left\langle\delta U^{2}\right\rangle r^{2} t}-1\right) .
$$

The basic relaxation mechanism is the same for the detuning as it is for the noisy pulses of finite width, so Eq. (20) bears a close similarity to Eq. (13). Notice also that $\bar{\epsilon}$ is not present in the formula (to this order in perturbation theory). When the pulse is turned off to let the qubit evolve on its own, it will be the bare energy $\epsilon$ that determines the qubit time $\tau_{q}$. Thus, the average variable $U$ does not appear in the results. There are two rates in Eq. (20). The first, $\Gamma_{\mathrm{D}}^{w}=r^{2}\left\langle\delta U^{2}\right\rangle$, is an additional contribution to the fast dephasing rate. The second is

$$
\Gamma_{w}=\frac{1}{r^{2}} \frac{\Delta^{2}}{\left\langle\delta U^{2}\right\rangle},
$$

which clearly corresponds to the slow nondemolition rate of the noisy pulses. The parameter $r^{-1}=\tau_{G} / \tau_{q}$ plays the role of the detuning parameter $\gamma$ in the previous section.

We now wish to combine all the phenomena discussed. When both the detuning $\gamma$ and the width of the pulses $r^{-1}$ are small, a double nondemolition measurement is taking place. In this case, we can write (in the vicinity 
of $\omega=0)$ an approximation to the spectral noise of the detector as a Lorentzian,

$$
\begin{aligned}
S(\omega) & =S_{q}+\frac{\lambda^{2}}{\pi} \frac{\Gamma}{\omega^{2}+\Gamma^{2}}, \quad \Gamma=\Gamma_{e}+\Gamma_{d}+\Gamma_{w}, \\
\Gamma_{d} & =\left(\frac{\gamma}{2 \pi}\right)^{2} \frac{2 \Delta^{2} \tau_{q}}{\left\langle\delta f^{2}\right\rangle}, \quad \Gamma_{w}=\left(\frac{1}{r}\right)^{2} \frac{\Delta^{2}}{\left\langle\delta U^{2}\right\rangle} .
\end{aligned}
$$

Here, the total broadening width of the spectral noise, $\Gamma$, has been decomposed into contributions from the environment, the detuning of the pulses from resonance, and the finite width of the pulses.

\section{IMPLEMENTATION WITH THE QUANTUM POINT CONTACT/DOUBLE DOT}

To see how this nondemolition measurement will work in practice, we illustrate it by using a double quantum dot (DD) as the qubit and the current through the Quantum Point Contact (QPC) as the measure-

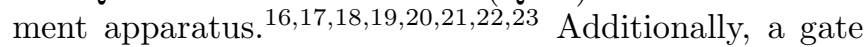
is placed above the QPC so that one may bias the barrier of the electrons in the QPC (Fig. 1). In this fashion, we can pulse the QPC so as to let current flow through during a short time when the gate voltage is raised above the barrier of the QPC. This pulsing is similar to the rectangular voltage pulses applied in the experiment of Ref. 24 and theoretically described in Ref. 25. The magnitude of this current will be sensitive to whether the electron is on the upper or lower part of the DD. At the detector output, there will be a current pulse train that may be analyzed statistically. The results of the nondemolition measurement will be seen in the spectral density of the current shot noise, Eq. (22).

The charge response time ${ }^{26} \tau_{R C}$ of a QPC is very fast. Here we assume that even the single particle dynamics of the QPC governed by $\tau_{\mathrm{QPC}}=\hbar /(\mathrm{eV})$ (where $V$ is the applied bias) is fast compared to the pulse duration $\tau_{G}$ which is in turn fast compared to the Rabi oscillation period of the qubit (we now restore the $\hbar$ for clarity). Therefore, a good separation of time scales may be reached for the pulse time $\tau_{G}$,

$$
\tau_{\mathrm{QPC}} \ll \tau_{G} \ll \tau_{q} .
$$

For solid state qubits, there is another constraint that true spins do not have: more states at higher energy. To avoid exciting the qubit into these states, a separate constraint is that $\tau_{G}>\hbar / \Delta E$, where $\Delta E$ is the energy difference to the next excited state. In this limit, we can use the physical setup described by Pilgram and one of the authors $\stackrel{21}{\underline{21}}$ The interaction Hamiltonian that couples the DD qubit to the detector is $H_{c}=\hat{Q}_{1} \hat{Q} / C_{i}$ where $\hat{Q}$ is the charge in the vicinity of the QPC, playing the role of $f$ and $\hat{Q}_{1}$ is charge on the DD. The current operator to be measured takes the form of a bare current $\hat{I}_{b}$ playing the role of $q$, plus a self-consistent screening current proportional to the the potential $\hat{U}$ of the QPC,

$$
\hat{I}=\hat{I}_{b}-\chi \hat{U}, \quad \chi=\frac{e^{2}}{2 \pi \hbar} \sum_{n} \frac{d T_{n}}{d E} e|V| .
$$

The (non-equilibrium) linear-response function $\chi$ is defined in terms of the (large) bias $V$ across the QPC, and the transmission probabilities of channel $n, T_{n}$. To express this in the form of Eq. (11), $\hat{U}$ must now be written in terms of the charge $\hat{Q}_{1}$ on the DD and the capacitances defined in Fig. $1, \hat{U}=\left[C_{p} /\left(C_{i} e^{2} D\right)\right] \hat{Q}_{1}$, where $D$ is the density of states at the Fermi energy in the scattering region, $C_{p}^{-1}=C_{1}^{-1}+C_{2}^{-1}+D^{-1}$ is the polarization capacitance, and $\hat{Q}_{1}=e \hat{\sigma}_{z}$. The deviation of the average current is given by

$$
\langle\Delta I(t)\rangle=\frac{\lambda}{2}\left\langle\sigma_{z}(t)\right\rangle,
$$

while the shot noise spectrum is given by

$$
S_{I I}(\omega)=S_{I_{b} I_{b}}+\frac{\lambda^{2}}{2} \tilde{K}(\omega)
$$

where $\tilde{K}(\omega)$ is the Fourier transform of $K(t)$. Thus, the response coefficient $\lambda$ is simply the difference in current output depending on whether the electron is in the upper or lower dot. In terms of the system parameters, it is given by

$$
\lambda=\frac{e C_{p}}{\pi \hbar C_{i} D} \sum_{n} \frac{d T_{n}}{d E} e|V| .
$$

Physically, $\lambda$ is proportional to the difference in transmission caused by the location of the DD electron, $\delta T=$ $(d T / d E) \delta E$, where $\delta E$ is the inverse density of states $D^{-1}$ and $C_{p} / C_{i}$ is the (small) coupling constant.

By doing an experiment where one varies the time between pulses and plots either the width of the line-shape of the spectral density or the signal-to-noise ratio as a function of the waiting time, the point of minimum width (or maximum signal-to-noise) finds both the oscillation time $\tau_{q}$ of the qubit as well as the system's natural (unmodified) relaxation rate. This is because of the fact that the width is simply a sum of the measurement backaction and uncontrolled environmental relaxation.

\section{CONCLUSIONS}

We have proposed a novel quantum nondemolition measurement on two-state systems with a kicking measurement device. By tuning the waiting time between kicks to be the oscillation period of the qubit dynamics, the weak measurement process does not act back on the desired operator being measured, though it does destroy the complimentary information in the conjugate variables. Deviations from this ideal scenario are taken into account by allowing for a detuning of the waiting time as 
well as for noisy pulses of finite width. These effects may be described by an approximate Lorentzian line-shape, whose width is the sum of three terms: the uncontrolled environmental relaxation rate, a QND detuning contribution, and a QND noisy pulse-width contribution. This novel QND proposal was illustrated with the quantum double-dot, measured by a gate-pulsed quantum point contact. The results of the nondemolition experiment may be obtained from the shot noise.

\section{ACKNOWLEDGMENTS}

We thank D. V. Averin and S. Pilgram for helpful discussions of their work. This work was supported by the Swiss National Science Foundation.

\section{APPENDIX A: PERTURBATION METHOD}

We now show how to obtain Eq. (20) from Eq. (18) and Eq. (19), considering the fact that the duration of the pulse is much shorter than one coherent oscillation. We use time-dependent perturbation theory by writing nested time ordered integrals to a given order, and then take averages over all the $\delta U$ 's. The primary object of interest is the correlator $K(t)=(1 / 2) \operatorname{Tr}\left[\sigma_{z}(t) \sigma_{z}(0)\right]$. At the initial time, $\operatorname{Tr}\left[\sigma_{x}(0) \sigma_{z}(0)\right]=\operatorname{Tr}\left[\sigma_{y}(0) \sigma_{z}(0)\right]=0$, so only the terms that involve $\sigma_{z}(0)$ will contribute to the correlator $K(t)$. We thus generate integral expressions for $\sigma_{z}(t)$ that end in $\sigma_{z}(0)$. As a simple example, the correlator to second order in time is found from

$$
\sigma_{z}(t)=\int_{0}^{t} d t^{\prime} \Delta \sigma_{y}\left(t^{\prime}\right)+\sigma_{z}(0)=\int_{0}^{t} d t^{\prime} \Delta\left\{\int_{0}^{t^{\prime}} d t^{\prime \prime}\left[\left(\bar{\epsilon}+r \delta U\left(t^{\prime \prime}\right)\right) \sigma_{x}\left(t^{\prime \prime}\right)-\Delta \sigma_{z}\left(t^{\prime \prime}\right)\right]+\sigma_{y}(0)\right\}+\sigma_{z}(0) .
$$

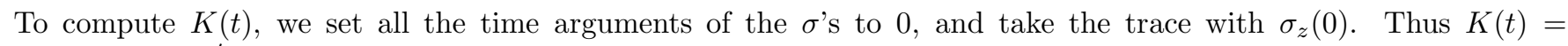
$1-\Delta^{2} \int_{0}^{t} d t^{\prime} \int_{0}^{t^{\prime}} d t^{\prime \prime}=1-\Delta^{2} t^{2} / 2$. To go to higher orders, the trick is to eliminate $\sigma_{x}$ :

$$
\sigma_{y}(t)=\sigma_{y}(0)+\int_{0}^{t} d t^{\prime}\left\{-\Delta \sigma_{z}\left(t^{\prime}\right)-\int_{0}^{t^{\prime}} d t^{\prime \prime}\left[\left(\bar{\epsilon}+r \delta U\left(t^{\prime}\right)\right)\left(\bar{\epsilon}+r \delta U\left(t^{\prime \prime}\right)\right)+\Delta^{2}\right] \sigma_{y}\left(t^{\prime \prime}\right)\right\} .
$$

The order of the expansion is given by the number of different time integrals. In general, there are many different terms to compute at each order. However, because the time $t$ is much shorter than the oscillation time, the dominate contribution is the one given by the maximal number of $r$ 's, or $\delta U$ 's. At order $2 n$, such terms are of the form $\Delta^{2} r^{2 n-2}$. To average over the fluctuating potentials, we use Wick's theorem to average the $2 n-2$ $\delta U$ 's in terms of $m=n-1$ pairwise contractions. We then compute average time ordered integrals of the form

$$
\int_{\left\{t^{\prime} s\right\}}\left\langle\delta U\left(t_{1}\right) \delta U\left(t_{2}\right) \ldots \delta U\left(t_{2 m}\right)\right\rangle
$$

In general, contractions of this form have every possible time-ordering of two $\delta U$ 's. However, the contrac- tion $\left\langle\delta U\left(t_{i}\right) \delta U\left(t_{j}\right)\right\rangle$ comes with a delta function enforcing $t_{i}=t_{j}$. If time-ordered integrals occur where $t_{i}$ and $t_{j}$ are not in adjacent time intervals, then this term is zero under the integration. The only term left will be the one where all time arguments are time ordered. Therefore, the integral Eq. (A3) is given by $\left\langle\delta U^{2}\right\rangle^{m} t^{m+2} /(m+2)$ !. We may now sum these dominate contributions at every order in time to obtain,

$$
\begin{aligned}
& K(t)=1-\frac{\Delta^{2} t^{2}}{2 !}+\sum_{n=2}^{\infty} \frac{(-1)^{n}}{(n+1) !}\left[\langle\delta U\rangle^{2} r^{2}\right]^{n-1} t^{n+1}, \\
& =1-\frac{\Delta^{2} t}{\left\langle\delta U^{2}\right\rangle r^{2}}-\frac{\Delta^{2}}{\left(\left\langle\delta U^{2}\right\rangle r^{2}\right)^{2}}\left(e^{-\left\langle\delta U^{2}\right\rangle r^{2} t}-1\right), \quad(\mathrm{A} 4)
\end{aligned}
$$

which is the same as Eq. (20).
1 M. Brune, S. Haroche, V. Lefevre, J. M. Raimond, and N. Zagury, Phys. Rev. Lett. 65, 976 (1990).

2 G. J. Pryde, J. L. O'Brien, A. G. White, S. D. Bartlett, and T. C. Ralph, Phys. Rev. Lett. 92, 190402 (2004).

3 V. B. Braginsky and F. Ya. Khalili, Rev. Mod. Phys. 68, 1 (1996).
${ }^{4}$ P. Grangier, J. A. Levenson, and J.-P. Poizat, Nature 396, 537 (1998).

5 T. A. Brun and H.-S. Goan, Phys. Rev. A 68, 032301 (2003).

${ }^{6}$ L. Bulaevskii, M. Hruška, A. Shnirman, D. Smith, and Yu. Makhlin, Phys. Rev. Lett. 92, 177001 (2004). 
7 D. V. Averin, Phys. Rev. Lett. 88, 207901 (2002).

${ }^{8}$ G. Casati, B. V. Chirikov, J. Ford, and F. M. Izrailev, Stochastic Behavior in Classical and Quantum Hamiltonian Systems Vol. 93 of Lecture Notes in Physics edited by G. Casati and J. Ford (Springer-Verlag: Berlin) p 334 (1979).

9 S. Fishman, D. R. Grempel, and R. E. Prange, Phys. Rev. Lett. 49, 509 (1982).

10 S. Fishman, I. Guarneri, and L. Rebuzzini, J. Stat. Phys. 110, 911 (2003).

11 S. Fishman, I. Guarneri, and L. Rebuzzini, Phys. Rev. Lett. 89, 084101 (2002).

12 K. S. Thorne, R. W. P. Drever, C. M. Caves, M. Zimmermann, and V. D. Sandberg, Phys. Rev. Lett. 40, 667 (1978).

13 V. B. Braginsky, Yu. I. Vorontsov, and F. Ya. Khalili, JETP Lett. 27, 276 (1978).

14 R. Ruskov, K. Schwab, and A. N. Korotkov, cond-mat/0406416

15 D. V. Averin, in "Exploring the Quantum-Classical Frontier: Recent Advances in Macroscopic and Mesoscopic Quantum Phenomena", Eds. J. R. Friedman and S. Han (Nova Science, Huntington, NY, 2003); cond-mat/0004364

16 A. A. Clerk, S. M. Girvin, and A. D. Stone, Phys. Rev. B 67, 165324 (2003).

17 A. N. Korotkov and D. V. Averin, Phys. Rev. B 64, 165310 (2001).

18 S. A. Gurvitz, Phys. Rev. B 56, 15215 (1997).

19 A. N. Korotkov, Phys. Rev. B 63, 115403 (2001).

${ }^{20}$ H. S. Goan and G. J. Milburn, Phys. Rev. B 64, 235307 (2001).

21 S. Pilgram and M. Büttiker, Phys. Rev. Lett. 89, 200401 (2002).

22 S. A. Gurvitz, L. Fedichkin, D. Mozyrsky, and G. P. Berman, Phys. Rev. Lett. 91, 066801 (2003).

23 A. A. Clerk and A. D. Stone, Phys. Rev. B 69, 24503 (2004).

24 T. Hayashi, T. Fujisawa, H.-Du Cheong, Y.-Ha Jeong, and Y. Hirayama, Phys. Rev. Lett. 91, 226804 (2003).

25 A. V. Pisliakov, M. F. Gelin, and W. Domcke, J. Phys. Chem. A 107, 2657 (2003).

26 T. Christen and M. Büttiker, Phys. Rev. Lett. 77, 143 (1996). 